\title{
The stellar initial mass function
}

\author{
Pavel Kroupa \\ Argelander-Institut für Astronomie, Universität Bonn, Auf dem Hügel 71, \\ D-53347 Bonn, Germany \\ email: pavel@astro.uni-bonn.de
}

\begin{abstract}
The IMF Universality Hypothesis cannot be discarded despite the existence of the Cluster IMF Theorem. This means that the currently existing star-formation theory fails to describe the stellar outcome. The IGIMF THEOREM, however, predicts a variation of galaxywide IMFs in dependence of the galaxy's star-formation rate even if the IMF UNIVERSALITY Hyротнеsis is valid. Taking indirect evidence from chemical evolution studies and the IGIMF THEOREM into account, it follows, however, that bulges and elliptical galaxies may have had a top-heavy IMF. A break-down of the IMF Universality THEOREM would thus be evident in extreme galaxy-wide $\left(\gtrsim 10 M_{\odot} /\right.$ yr $)$ star-formation events.
\end{abstract}

Keywords. stars: luminosity function, mass function - binaries: general - stars: formation open clusters and associations: general - Galaxy: stellar content - Galaxy: evolution - galaxies: stellar content - galaxies: evolution - galaxies: star clusters

\section{Introduction}

The stellar initial mass function (IMF), $\xi(m) d m$, where $m$ is the stellar mass, is the parent distribution function of the masses of stars formed in one event. Here, the number of stars in the mass interval $m, m+d m$ is $d N=\xi(m) d m$. The IMF is, strictly speaking, a hypothetical construct because any observed system of $N$ stars merely constitutes a particular representation of this universal distribution function (Elmegreen 1997; Maíz Apellániz \& Úbeda 2005). The probable existence of a unique $\xi(m)$ can be inferred from observations of an ensemble of systems each consisting of $N$ stars (e.g. Massey 2003). If, after corrections for (a) stellar evolution, (b) unknown multiple stellar systems, and (c) stellar-dynamical biases, the individual distributions of stellar masses is similar within the statistical uncertainties, then we (the community) deduce that the hypothesis that the stellar mass distributions are not the same can be excluded. That is, we make the case for a universal, standard or canonical stellar IMF within the physical conditions probed by the relevant physical parameters (metallicity, density, mass) of the populations at hand.

\section{The canonical or standard form of the stellar IMF}

The canonical stellar IMF is a two-part-power law, $\xi(m) \propto m^{-\alpha_{i}}$, the only structure with confidence found so far being the change of index from the Salpeter/Massey value to a smaller one near $0.5 M_{\odot} \dagger$ :

$$
\begin{aligned}
\alpha_{1}=1.3 \pm 0.3, & 0.08 \lesssim m / M_{\odot} \lesssim 0.5, \\
\alpha_{2}=2.3 \pm 0.5, & 0.5 \lesssim m / M_{\odot} \lesssim 150 .
\end{aligned}
$$

It has been corrected for bias through unresolved multiple stellar systems in the lowmass $\left(m<1 M_{\odot}\right)$ regime using a multi-dimensional optimisation technique. The general

$\dagger$ The uncertainties in $\alpha_{i}$ are estimated from the alpha-plot ( $\left.\S 3.2\right)$, as shown in fig. 5 in Kroupa (2002), to be about $95 \%$ confidence limits. 
outline of this technique is as follows (Kroupa et al. 1993): first the correct form of the stellar-mass-luminosity relation is extracted using observed stellar binaries and theoretical constraints on the location, amplitude and shape of the minimum of its derivative near $m=0.3 M_{\odot}, M_{V} \approx 12, M_{I} \approx 9$ in combination with the observed shape of the nearby and deep Galactic-field LF. Having established the semi-empirical mass-luminosity relation of stars, which is an excellent fit to the most recent observational constraints by Delfosse et al. (2000), a model of the Galactic field is then calculated assuming a parametrised form for the MF and different values for the scale-height of the Galactic-disk, and different binary fractions in it. Measurement uncertainties and age and metallicity spreads must also be considered in the theoretical stellar population. Optimisation in this multi-parameter space (MF parameters, scale-height and binary population) against observational data leads to the canonical stellar MF for $m<1 M_{\odot}$. A consistency-check is then performed as follows: the above MF is used in creating young populations of binary systems that are born in modest star clusters consisting of a few hundred stars. Their dissolution into the Galactic field is computed with an $N$-body code, and the resulting theoretical field is compared to the observed LFs (Kroupa 1995a,b). Further confirmation comes from independent sources, most notably by Reid, Gizis \& Hawley (2002) and also Chabrier (2003).

In the high-mass regime, Massey (2003) reports the same slope or index $\alpha_{3}=2.3 \pm 0.1$ for $m \gtrsim 10 M_{\odot}$ in many $\mathrm{OB}$ associations and star clusters in the Milky Way (MW), the Large- and Small-Magellanic clouds (LMC, SMC, respectively). It is therefore suggested to refer to $\alpha_{2,3}=2.3$ as the Salpeter/Massey slope or index, given the pioneering work of Salpeter (1955) who derived this value for stars with masses $0.4-10 M_{\odot}$. However, multiplicity corrections await to be done once we learn more about how the components are distributed in massive stars (cf. Preibisch et al. 1999; Zinnecker 2003). Scalo (1986) found $\alpha_{\text {MWdisk }} \approx 2.7(m \gtrsim 1 M \odot)$ from a very thorough analysis of OB star counts in the MW disk. Similarly, the star-count analysis of Reid et al. (2002) leads to $2.5 \lesssim \alpha_{\text {MWdisk }} \lesssim 2.8$, and Tinsley (1980), Kennicutt (1983; his "extended Miller-Scalo IMF"), Portinari et al. (2000) and Romano et al. (2005) find $2.5 \lesssim \alpha_{\mathrm{MWdisk}} \lesssim 2.7$. That $\alpha_{\mathrm{MWdisk}}>\alpha_{2}$ naturally is shown in $\S 4$.

The evidence for a universal upper mass cutoff near $150 M_{\odot}$ (Weidner \& Kroupa 2004; Figer 2005; Oey \& Clarke 2005; Koen 2006; Maíz Apellániz 2007) seems to be rather well established in populations with metallicities ranging from the LMC $(Z \approx 0.008)$ to the super-solar Galactic centre $(Z \gtrsim 0.02)$ such that the stellar mass function (MF) simply stops at that mass. This mass needs to be understood theoretically (see discussion in Kroupa \& Weidner 2005).

Below the hydrogen-burning limit there is substantial evidence that the IMF flattens further to $\alpha \approx 0.3 \pm 0.5$ (Martín et al. 2000; Chabrier 2003; Moraux et al. 2004). Therefore, the canonical IMF most likely has a peak at $0.08 M_{\odot}$. Brown dwarfs, however, comprise only a few $\%$ of the mass of a population and are therefore dynamically irrelevant. The logarithmic form of the canonical IMF, $\xi_{\mathrm{L}}(m)=\ln (10) m \xi(m)$, which gives the number of stars in $\log _{10} m$-intervals, also has a peak near $0.08 M_{\odot}$. However, the system IMF (of stellar single and multiple systems combined to system masses) has a maximum in the mass range $0.4-0.6 M_{\odot}$ (Kroupa et al. 2003).

The above canonical or standard form has been derived from detailed considerations of star-counts thereby representing an average IMF: for low-mass stars it is a mixture of stellar populations spanning a large range of ages $(0-10 \mathrm{Gyr})$ and metallicities $([\mathrm{Fe} / \mathrm{H}] \gtrsim-1)$. For the massive stars it constitutes a mixture of different metallicities $([\mathrm{Fe} / \mathrm{H}] \gtrsim-1.5)$ and star-forming conditions (OB associations to very dense star-burst clusters: R136 in the LMC). Therefore it can be taken as a canonical form, and the aim is to test the 
IMF Universality Hypothesis: the canonical IMF constitutes the parent distribution of all stellar populations.

Negation of this hypothesis would imply a variable IMF. Note that the work of Massey (2003) has already established the IMF to be invariable for $m \gtrsim 10 M_{\odot}$ and for densities $\rho \lesssim 10^{5}$ stars $/ \mathrm{pc}^{3}$ and metallicity $Z \gtrsim 0.002$.

\section{Universality of the IMF: resolved populations}

The strongest test of the IMF UnIVERSALITy Hypothesis (p. 111) is obtained by studying populations that can be resolved into individual stars. Since one also seeks co-eval populations at the same distance and with the same metallicity to minimise uncertainties, star clusters and stellar associations would seem to be the test objects of choice. But before contemplating such work some lessons from stellar dynamics are useful:

\subsection{Star clusters and associations}

To access a pristine population one would consider observing star-clusters that are younger than a few Myr. However, such objects carry rather massive disadvantages: the pre-main sequence stellar evolution tracks are unreliable (Baraffe et al. 2002; Wuchterl \& Tscharnuter 2003) such that the derived masses are uncertain by at least a factor of about two. Remaining gas and dust lead to patchy obscuration. Very young clusters evolve rapidly: the dynamical crossing time is $t_{\mathrm{cr}}=2 r_{\mathrm{cl}} / \sigma_{\mathrm{cl}}$, where the cluster radii are typically $r_{\mathrm{cl}}<1 \mathrm{pc}$ and for cluster masses $M_{\mathrm{cl}}>10^{3} M_{\odot} \sigma_{\mathrm{cl}}>2 \mathrm{~km} / \mathrm{s}$ such that $t_{\mathrm{cr}}<1 \mathrm{Myr}$. The inner regions of populous clusters have $t_{\mathrm{cr}} \approx 0.1 \mathrm{Myr}$, and thus significant mixing and relaxation (relaxation time $t_{\text {relax }} \approx t_{\mathrm{cr}} 0.1 \mathrm{~N} / \ln N$ ) occurs there by the time the residual gas is expelled by the winds and photo-ionising radiation from massive stars, if they are present $(N \gtrsim 1000)$. Much of this is summarised in Kroupa (2005). For example, the $0.5-2$ Myr old Orion Nebula cluster (ONC), which is known to be supervirial with a virial mass about twice the observed mass (Hillenbrand \& Hartmann 1998), has already expelled its residual gas and is expanding rapidly thereby probably having lost its outer stars (Kroupa, Aarseth \& Hurley 2001). The super-virial state of young clusters makes measurements of their mass-to-light ratia a bad measure of the stellar mass within them (Bastian \& Goodwin 2006; Goodwin \& Bastian 2006), and rapid dynamical mass-segregation likewise makes "naive" measurements of the $M / L$ ratia wrong (Boily et al. 2005; Fleck et al. 2006).

Massive stars $\left(m>8 M_{\odot}\right)$ are either formed at the cluster centre or get there through dynamical mass segregation, i.e. energy equipartition (Bonnell, Larson \& Zinnecker 2006). The latter process is very rapid and can occur within 1 Myr. A cluster core of massive stars is therefore either primordial or forms rapidly because of energy equipartition in the cluster, and it is dynamically highly unstable decaying within a few $t_{\text {cr, core }}$. The ONC, for example, should not be hosting a Trapezium as it is extremely volatile. The implication for the IMF is that the ONC and other similar clusters and the OB associations which stem from them must be very depleted in their massive-star content (Pflamm-Altenburg \& Kroupa 2006a).

Corrections for the high multiplicity fraction of the very young population are very uncertain because the binary population is in a state of change (Kroupa 2001; Kroupa et al. 2001).

The determination of an IMF relies on the assumption that all stars in a very young cluster formed together. Trapping of older field or $\mathrm{OB}$ association stars by the 
forming cluster has been found to be possible for ONC-type clusters (Pflamm-Altenburg $\&$ Kroupa 2006b) and also for massive $\omega$-Cen type clusters (Fellhauer, Kroupa \& Evans 2006). Additionally, the sample of cluster stars may be contaminated by enhanced foreand back-ground densities of field stars due to focussing of stellar orbits during cluster formation (Pflamm-Altenburg \& Kroupa 2006b).

Thus, be it at the low-mass end or the high-mass end, the "IMF" estimated from very young clusters cannot be the true IMF. Statistical corrections for the above effects need to be applied, and for this comprehensive $N$-body modelling is required.

Old open clusters in which most stars are on or near the main sequence are no better stellar samples: They are dynamically highly evolved, since they have left their previous concentrated and gas-rich state and so they contain only a fraction of the stars originally born in the cluster (Kroupa \& Boily 2002; Weidner et al. 2007; Baumgardt \& Kroupa 2007). The binary fraction is typically high and comparable to the Galactic field, but does depend on the initial density and the age of the cluster, the mass-ratio distribution of companions also. So, simple corrections cannot be applied equally for all old clusters. The massive stars have died, and secular evolution begins to affect the remaining stellar population (after gas expulsion) through energy equipartition. Baumgardt \& Makino (2003) have quantified the changes of the MF for clusters of various masses and on different Galactic orbits. Near the half-mass radius the local MF resembles the global MF in the cluster, but the global MF becomes significantly depleted of its lesser stars already by about $20 \%$ of the cluster's disruption time.

Given that we are never likely to learn the exact dynamical history of a particular cluster, it follows that we can never ascertain the IMF for any individual cluster.

The above can be summarised concisely with the following theorem:

Cluster IMF Theorem: The IMF cannot be extracted for any individual star cluster.

PRoOF: For clusters younger than about $0.5 \mathrm{Myr}$ star formation has not ceased and the IMF is therefore not assembled yet and the cluster cores consisting of massive stars have already dynamically ejected members (Pflamm-Altenburg \& Kroupa 2006). For clusters with an age between 0.5 and a few Myr the expulsion of residual gas has lead to loss of stars (Kroupa et al. 2001). Older clusters are either still loosing stars due to residual gas expulsion or are evolving secularly through evaporation driven by energy equipartition (Baumgardt \& Makino 2003). There exists thus no time when all stars are assembled in an observationally accessible volume (i.e. a star cluster). End of proof.

Note that the Cluster IMF THEOREM implies that individual clusters cannot be used to make deductions on the similarity or not of their IMFs, unless a complete dynamical history of each cluster is available.

Notwithstanding this pessimistic theorem, it is nevertheless necessary to observe and study star clusters of any age. Combined with thorough and realistic $N$-body modelling the data do lead to essential statistical constraints on the IMF UNIVERSALITY HYPOTHESIS (p. 111). Such an approach is discussed in the next section.

\subsection{The alpha plot}

Scalo (1998) conveniently summarised a large part of the available observational constraints on the IMF of resolved stellar populations with the alpha plot, as used by Kroupa (2001, 2002) for explicit tests of the IMF Universality HyPothesis (p. 111) given the Cluster IMF Theorem (p. 112). One example is presented in Fig. 1, which demonstrates that the observed scatter in $\alpha(m)$ can be readily understood as being due to 


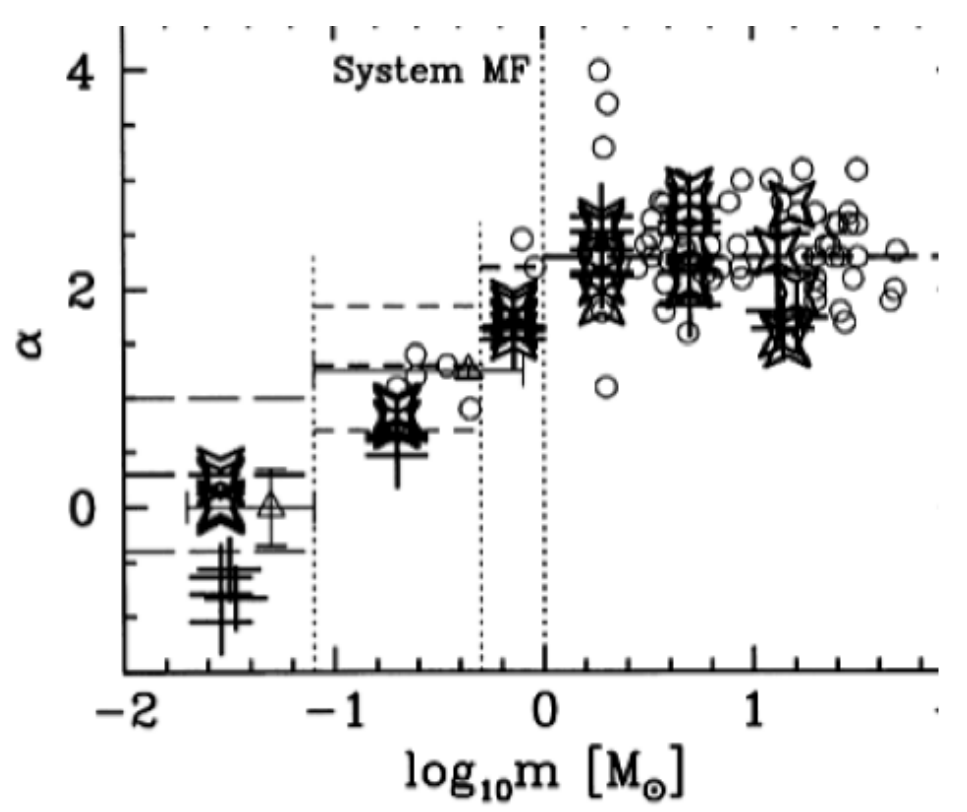

Figure 1. The alpha plot. Open circles are the observational constraints for open clusters and associations for the MW, Large and Small Magellanic clouds collated mostly by Scalo (1998). The open stars (crosses) are theoretical star clusters "observed" in the computer at an age of 3 (0) Myr and within a radius of $3.2 \mathrm{pc}$ from the cluster centre. The 5 clusters have 3000 stars in 1500 binaries initially and the assumed IMF is the canonical one. The theoretical data nicely show a similar spread as the observational ones; note the binary-star-induced depression of $\alpha_{2}$ in the mass range $0.1-0.5 M_{\odot}$ - the theoretical data reproduce more-recent data shown in Kroupa (2002) very well. The IMF UnIVERSALITy Hypothesis can therefore not be discarded given the observational data. Models from Kroupa (2001).

Poisson uncertainties (see also Elmegreen 1997, 1999) and dynamical effects, as well as arising from biases through unresolved multiple stars. Furthermore, there is no evident systematic change of $\alpha$ at a given $m$ with metallicity or density of the star-forming cloud, so that the IMF UNIVERSALITY HyPothesis cannot be falsified.

\subsection{Very ancient resolved populations}

Witnesses of the early formation phase of the MW are its globular clusters. Such $10^{4-6} M_{\odot}$ clusters formed with individual star-formation rates of $0.1-1 M_{\odot} / \mathrm{yr}$ and densities $\approx 5 \times 10^{3-5} M_{\odot} / \mathrm{pc}^{3}$. These are relatively high values, when compared with the current star-formation activity in the MW disk. For example, a $5 \times 10^{3} M_{\odot}$ Galactic cluster forming in $1 \mathrm{Myr}$ corresponds to a star formation rate of $0.005 M_{\odot} / \mathrm{yr}$. The alpha plot, however, does not support any significant systematic difference between the IMF of stars formed in globular clusters and present-day low-mass star formation. For massive stars, it can be argued that the mass in stars more massive than $8 M_{\odot}$ cannot have been larger than about half the cluster mass, because otherwise the globular clusters would not be as compact as they are today. This constrains the IMF to have been close to the canonical IMF (Kroupa 2001).

A particularly exotic star-formation mode is thought to have occurred in dwarf-spheroidal (dSph) satellite galaxies. The MW has about 19 such satellites at distances from 50 to $250 \mathrm{kpc}$ (Metz \& Kroupa 2007). These objects have stellar masses and ages comparable to those of globular clusters but are $10-100$ times larger and are thought 
to have 10 - 100 times more mass in dark matter than in stars. They also show evidence for complex star-formation activity and metal-enrichment histories and must have therefore formed under rather exotic conditions. Nevertheless, the MFs in two of these satellites are found to be indistinguishable from those of globular clusters in the mass range $0.5-0.9 M_{\odot}$, thus again showing consistency with the canonical IMF (Grillmair et al. 1998; Feltzing, Gilmore \& Wyse 1999).

\section{Composite populations: the IGIMF}

The vast majority of all stars form in embedded clusters and so the correct way to proceed to calculate a galaxy-wide stellar IMF is to add-up all the IMFs of all starclusters born in one "star-formation epoch". Such "epochs" may be identified with the Zoccali et al. star-burst events creating the Bulge. In disk galaxies they may be related to the time-scale of transforming the inter-stellar matter to star clusters along spiral arms. Addition of the clusters born in one epoch gives the integrated galactic initial mass function, the IGIMF (Kroupa \& Weidner 2003).

IGIMF DEFInITION: The IGIMF is the IMF of a composite population which is the integral over a complete ensemble of simple stellar populations.

Note that a simple population has a mono-metallicity and a mono-age distribution and is therefore a star cluster. A "complete ensemble" is a statistically complete representation of the initial cluster mass function (ICMF) in the sense that the actual mass function of $N_{\mathrm{cl}}$ clusters lies within the expected statistical variation of the ICMF.

IGIMF TheOrEm: The IGIMF is not equal to the canonical IMF.

ProOF: Weidner \& Kroupa (2006) calculate that the IGIMF is steeper than the canonical IMF for $m \gtrsim 1 M_{\odot}$ if the IMF UnIVERSALITY Hypothesis holds. The steepening becomes negligible if the power-law ICMF is flatter than $\beta \approx 1$.8. End of proof.

It may be argued that IGIMF $=$ IMF (e.g. Elmegreen 2006) because when a star cluster is born, its stars are randomly sampled from the IMF up to the most massive star possible. On the other hand, the physically-motivated ansatz by Weidner \& Kroupa (2005, 2006) of taking the mass of a cluster as the constraint and of including the observed correlation between the maximal star mass and the cluster mass, yields an IGIMF which is equal to the canonical IMF for $m \lesssim 1.5 M_{\odot}$ but which is systematically steeper above this mass. By incorporating the observed maximal-cluster-mass vs star-formation rate of galaxies for the youngest clusters (Weidner, Kroupa \& Larsen 2004), it follows for $m \gtrsim 1.5 M_{\odot}$ that low-surface-brightness (LSB) galaxies have very steep IGIMFs while normal or $\mathrm{L}_{*}$ galaxies have Scalo-type IGIMFs. This systematic shift of $\alpha_{\text {IGIMF }}\left(m \gtrsim 1.5 M_{\odot}\right)$ with galaxy type implies that less-massive galaxies have a significantly suppressed supernova II rate per low-mass star. They also show a slower chemical enrichment such that the observed metallicity-galaxy-mass relation can be nicely accounted for (Koeppen, Weidner \& Kroupa 2007).

It is striking that Lee et al. (2004) have indeed found LSBs to have bottom-heavy IMFs, while Portinari et al. (2004) and Romano et al. (2005) find the MW disk to have a steeper-than Salepeter IMF for massive stars which is, in comparison with Lee et al., much flatter than the IMF of LSBs, as required by the IGIMF THEOREM. 


\section{The Galactic Bulge and other ellipticals - a clear case for top-heavy IGIMFs?}

Based on photo-chemical evolution studies Romano et al. (2005) show the MW disk to be reproducible by a Galactic-field stellar IMF with a Scalo slope above $1 M_{\odot}\left(\alpha_{3} \approx 2.7\right)$ rather than with the canonical IMF (eq. 2.1). This is supported by the independent work of Portinari, Sommer-Larsen \& Tantalo (2004).

In contrast, the MW Bulge may require a rapid phase of formation, lasting $\lesssim 0.1 \mathrm{Gyr}$, and a top-heavy Bulge-wide IMF with $\alpha_{3} \approx 1.95$ (Ballero, Matteucci \& Origlia 2006). For elliptical galaxies a similar scenario seems to hold, whereby the galaxy-wide IMF needed to account for the colours and chemical properties is the canonical one $\left(\alpha_{3} \approx 2.3\right)$, but flattening (decreasing $\alpha_{3}$ ) slightly with increasing galaxy mass (Pipino \& Matteucci 2004). An experiment, where the yields for massive stars are changed as much as possible without violating available constraints confirms this result - it seems not to be possible to understand the metallicity distribution of MW Bulge stars with a Galactic field IMF (i.e. with $\alpha_{3}=\alpha_{\text {Scalo }}=2.7$ ). Instead, $\alpha_{3} \approx 1.9$ seems to be required (Ballero, Kroupa \& Matteucci 2007). Zoccali et al. (2006) find the "MW bulge to be similar to early-type galaxies, in being $\alpha$-element enhanced, dominated by old stellar populations, and having formed on a timescale shorter than $\approx 1$ Gyr". "Therefore, like early-type galaxies the MW Bulge is likely to have formed through a short series of star-bursts triggered by the coalescence of gas-rich mergers, when the universe was only a few Gyr old". For lowmass stars, Zoccali et al. (2000) find the Bulge to have a similar MF as the MW disk and globular clusters.

It thus appears that the system-wide IMF for Bulges and massive ellipticals is topheavy. According to the IGIMF THEOREM below this can only be the case if the IMF Universality Hypothesis breaks down. Given the reported short formation timescales of the Bulge and massive ellipticals of $0.1-1$ Gyr, it follows that the IMF UNIVERSALITY HyPOTHESIS is invalid when star-formation rates in excess of about $10 \mathrm{M} \odot / \mathrm{yr}$ are reached.

\section{Origin of the IMF: theory vs observations}

General physical concepts such as coalescence of proto-stellar cores, mass-dependent focussing of gas accretion onto proto-stars, stellar feedback, and fragmentation of molecular clouds lead to predictions of systematic variations of the IMF with changes in the physical conditions of star formation (Murray \& Lin 1996; Elmegreen 2004; Tilley \& Pudritz 2005; Casuso \& Beckman 2007). The IMF is shown to vary, for example becoming top-heavy with decreasing metallicity or increasing temperature of the cloud. Thus, the thermal Jeans mass of a molecular cloud decreases with temperature and increasing density, implying that for higher metallicity (= stronger cooling) and density the IMF should shift on average to smaller stellar masses (e.g. Larson 1998; Bonnell, Larson \& Zinnecker 2006). The entirely different notion that stars regulate their own masses through a balance between feedback and accretion also implies smaller stellar masses for higher metallicity due in part to more dust and thus more efficient radiation pressure (Adams \& Fatuzzo 1996; Adams \& Laughlin 1996).

Klessen, Spaans \& Jappsen (2007) report state-of-the art calculations of star-formation under physical conditions as found in molecular clouds near the Sun and they are able to reproduce the canonical IMF. Applying the same computational technology to the conditions near the Galactic centre they obtain a theoretical IMF in agreement with the previously reported apparent decline of the stellar MF in the Arches cluster below about 


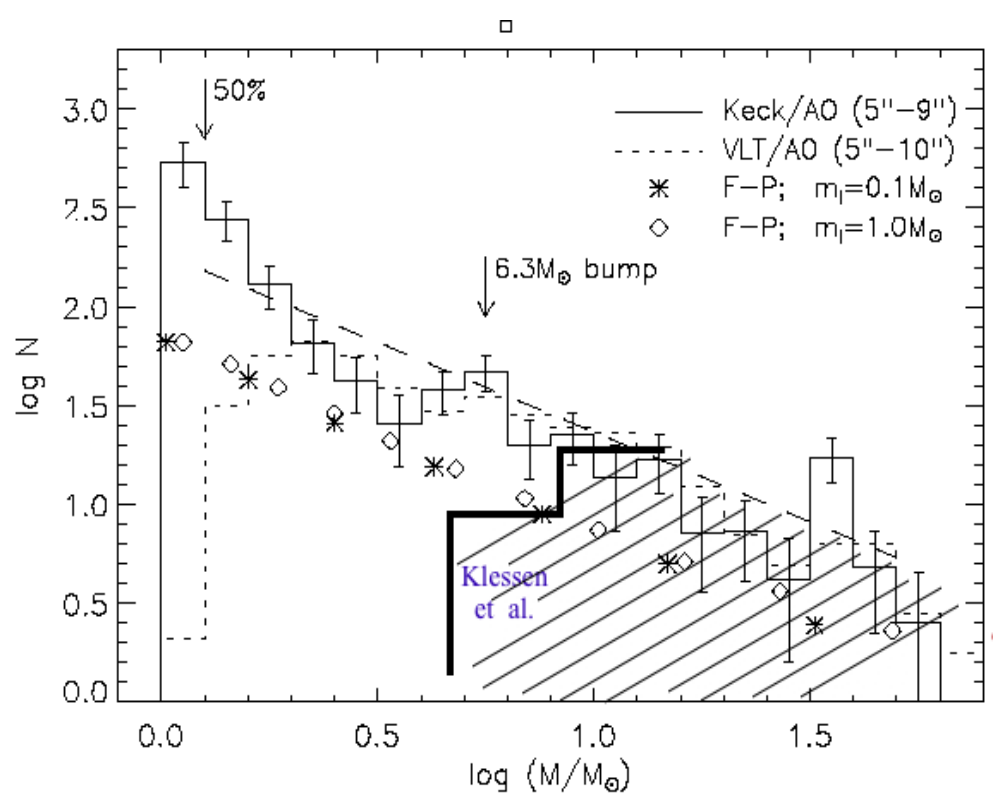

Figure 2. The observed mass function of the Arches cluster near the Galactic centre by Kim et al. (2007) shown as the thin histograms is confronted with the theoretical MF for this object calculated with the SPH technique by Klessen et al. (2007). The latter has a down-turn incompatible to the observations therewith ruling out a theoretical understanding of the stellar mass spectrum ("one counter-example suffices to bring-down a theory"). One possible reason for the theoretical failure may be the assumed turbulence driving. For details on the figure see Kim et al. (2007).

$6 M_{\odot}$. Kim et al. (2007) published their observations of the Arches cluster shortly after Klessen et al. (2007) and performed the necessary state-of-the art $N$-body calculations of the dynamical evolution of this young cluster, revising our knowledge significantly. They find that the MF continues to increase down to their $50 \%$ completeness limit $\left(1.3 M_{\odot}\right)$ with a power-law exponent only slightly shallower than the canonical Massey/Salpeter value once mass-segregation is corrected for. This situation is demonstrated in Fig. 2.

The observations, on the other hand side, appear to suggest that the canonical IMF is frozen-in already at the pre-stellar cloud-core level (Motte et al. 1998; 2001). Nutter \& Ward-Thompson (2007) and Alves, Lombardi \& Lada (2007) find, however, the prestellar cloud cores to be distributed according to the same shape as the canonical IMF, but shifted to larger masses by a factor of about three or more. This is suggested to perhaps mean a star-formation efficiency per star of $30 \%$ or less independently of stellar mass. The interpretation of such observations in view of multiple star formation in each cloud-core is being studied by Goodwin et al. (2007).

\section{Conclusions}

The IMF Universality Hypothesis, the Cluster IMF Theorem and the IGIMF THEOREM have been stated. Furthermore,

(a) The stellar luminosity function has a pronounced maximum at $M_{V} \approx 12$ which is universal and well understood as a result of stellar physics. Thus by counting stars on the sky we can look into their interiors. 
(b) Unresolved multiple systems must be accounted for when the MFs of different stellar populations are compared.

(c) The canonical IMF (eq. 2.1) fits the solar-neighbourhood star counts and all resolved stellar populations available to-date, with one exception (point $h$ below).

(d) Simple stellar populations are to be found in individual star clusters with $M_{\mathrm{cl}}$ $\lesssim 10^{6} M_{\odot}$. These have the canonical IMF.

(e) Composite populations describe entire galaxies. They are a result of many epochs of star-cluster formation and are described by the IGIMF THEOREM.

$(f)$ The IGIMF above $\approx 1 M_{\odot}$ is steep for LSB galaxies, flattening to the Scalo slope $\left(\alpha_{\text {IGIMF }} \approx 2.7\right)$ for $L_{*}$ disk galaxies. This is nicely consistent with the IMF UNIVERSALITY HYPOTHESIS in the context of the IGIMF THEOREM.

(g) Therefore, the IMF Universality Hypothesis can not be excluded despite the CLUSTER IMF THEOREM for conditions $\rho \lesssim 10^{5}$ stars $/ \mathrm{pc}^{3}$ and $Z \gtrsim 0.002$.

(h) Further flattening of the IGIMF to massive spheroids (the MW Bulge and elliptical galaxies) to $\alpha_{\text {IGIMF }} \approx 1.9$ is suggested by a number of independent but indirect studies. This empirical result is qualitatively consistent with the IGIMF THEOREM, but only if the IMF UNIVERSALITY HYPOTHESIS is invalid for extreme star-burst conditions.

(i) Modern star-formation computations appear to give wrong results concerning the shape of the stellar IMF.

(j) The stellar IMF appears to be frozen-in at the pre-stellar cloud-core stage therewith probably being a result of the processes leading to the formation of self-gravitating molecular clouds.

\section{Acknowledgements}

I am very grateful to the organisers for a most enjoyable meeting. I also thank Thomas Maschberger and Jan Pflamm-Altenburg for helpful discussions and comments. Parts of this work were supported by the DFG.

\section{References}

Adams, F. C., \& Fatuzzo, M. (1996). ApJ 464, 256

Adams, F. C., \& Laughlin, G. (1996). ApJ, 468, 586

Alves, J., Lombardi, M., \& Lada, C. J. (2007). A $\& A$, 462, L17

Ballero, S., Matteucci, F., \& Origlia, L. (2006). astro-ph/0611650

Ballero, S., Kroupa, P., \& Matteucci, F. (2007), MNRAS, submitted

Baraffe, I., Chabrier, G., Allard, F., \& Hauschildt, P. H. (2002). A $\& A$, 382, 563

Baumgardt, H., \& Kroupa, P., (2007). MNRAS, submitted

Baumgardt, H., \& Makino, J. (2003). MNRAS 340, 227

Bastian, N., \& Goodwin, S. P. (2006). MNRAS, 369, L9

Boily, C. M., Lançon, A., Deiters, S., \& Heggie, D. C. (2005). ApJ, 620, L27

Bonnell, I. A., \& Davies, M. B. (1998). MNRAS 295, 691

Bonnell, I. A., Larson, R., \& Zinnecker, H. (2006) Proto Stars and Planets V, astro-ph/0603447

Casuso, E., \& Beckman, J.E., (2007). ApJ in press

Chabrier, G. (2003). PASP 115, 763

Delfosse, X., Forveille, T., Ségransan, D., Beuzit, J.-L., Udry, S., Perrier, C., \& Mayor, M. (2000). $A \& A$ 364, 217

Elmegreen, B. G. (1997). ApJ 486, 944

Elmegreen, B. G. (1999). ApJ 515, 323

Elmegreen, B. G. (2004). MNRAS, 354, 367

Elmegreen, B. G. (2006). ApJ 648, 572

Fellhauer, M., Kroupa, P., \& Evans, N. W. (2006). MNRAS 372, 338

Feltzing, S., Gilmore, G., \& Wyse, R. F. G. (1999). ApJ, 516, L17 
Figer, D. F. (2005). Nature 434, 192

Fleck, J.-J., Boily, C. M., Lançon, A., \& Deiters, S. (2006). MNRAS, 369, 1392

Goodwin, S. P., \& Bastian, N. (2006). MNRAS 373, 752

Goodwin, A. P., Nutter, D., \& Kroupa, P., Ward-Thompson, D., Whitworth, A.P., (2007). in preparation

Grillmair, C. J., et al. (1998). AJ, 115, 144

Hillenbrand, L. A., \& Hartmann, L. W. (1998). ApJ 492, 540

Kennicutt, R. C., Jr. (1983). ApJ 272, 54

Kim, S. S., Figer, D. F., Kudritzki, R. P., \& Najarro, F. (2006). ApJL, astro-ph/0611377

Klessen, R. S., Spaans, M., \& Jappsen, A.-K. (2006). MNRASL115, astro-ph/0610557

Koen, C. (2006). MNRAS 365, 590

Koeppen, J., Weidner, C., \& Kroupa, P. (2007). MNRAS, in press, astro-ph/0611723

Kroupa, P., (1995a). ApJ 453, 350

Kroupa, P. (1995b). ApJ 453, 358

Kroupa, P. (2001). MNRAS 322, 231

Kroupa P. (2002). Science 295, 82

Kroupa, P. (2005), in ESA SP-576: The Three-Dimensional Universe with Gaia, 629 (astro$\mathrm{ph} / 0412069)$

Kroupa, P., \& Boily, C. M. (2002). MNRAS 336, 1188

Kroupa, P., \& Weidner, C. (2003). ApJ 598, 1076

Kroupa, P., Weidner, C. (2005). IAU Symposium 227, 423, astro-ph/0507582

Kroupa, P., Aarseth, S., \& Hurley, J. (2001). MNRAS 321, 699

Kroupa, P., Tout, C. A., \& Gilmore, G. (1993). MNRAS 262, 545

Kroupa, P., Bouvier, J., Duchêne, G., \& Moraux, E. (2003). MNRAS 346, 354

Larson, R. B. (1998). MNRAS, 301, 569

Lee, H.-c., Gibson, B.K., Flynn, C., Kawata, D., \& Beasley, M.A. (2004). MNRAS 353, 113

Maíz, Apellániz, J. (2007). ApJ submitted (astro-ph/0612012)

Maíz Apellániz, J., \& Úbeda, L. (2005). ApJ 629, 873

Martín, E. L., Brandner, W., Bouvier, J., Luhman, K. L., Stauffer, J., Basri, G., Zapatero Osorio, M. R., \& Barrado, y Navascués, D. (2000). ApJ 543, 299

Massey P. (2003). AREA 41, 15

Metz, M., \& Kroupa, P. (2007). MNRAS astro-ph/0701289

Moraux, E., Bouvier, J., \& Clarke, C. (2004). SF2A-2004: Semaine de l'Astrophysique Francaise, F. Combes, D. Barret, T. Contini, F. Meynadier and L. Pagani (eds), 251

Motte, F., Andre, P., \& Neri, R. (1998). A\&\&A 336, 150

Motte, F., André, P., Ward-Thompson, D., \& Bontemps, S. (2001). A\&GA 372, L41

Murray, S. D., \& Lin, D. N. C. (1996). ApJ 467, 728

Nutter, D., \& Ward-Thompson, D. (2007). MNRAS, in press astro-ph/0611164

Oasa, Y., et al.

Oey, M. S., \& Clarke, C. J. (2005). ApJL 620, L43

Pflamm-Altenburg, J., \& Kroupa, P. (2006a). MNRAS 373, 295

Pflamm-Altenburg, J., \& Kroupa, P. (2006b). MNRAS in press, astro-ph/0611517

Pipino, A., \& Matteucci, F. (2004). MNRAS 347, 968

Portinari, L., Sommer-Larsen, J., \& Tantalo, R. (2004). MNRAS 347, 691

Preibisch, T., Balega, Y., Hofmann, K., Weigelt, G., \& Zinnecker, H. (1999). New Astronomy 4, 531

Reid, I. N., Gizis, J. E., \& Hawley, S. L. (2002). AJ 124, 2721

Romano, D., Chiappini, C., Matteucci, F., \& Tosi, M. (2005). A $\& A$ 430, 491

Salpeter, E. E. (1955). ApJ 121, 161

Scalo, J. M. (1986). Fundamentals of Cosmic Physics 11, 1

Scalo, J. (1998). in ASP Conf. Ser. 142: The Stellar Initial Mass Function (38th Herstmonceux Conference) The IMF Revisited: A Case for Variations. pp. 201

Tilley, D. A., \& Pudritz, R. E. (2005). Protostars and Planets V, 8473

Tinsley, B. M. (1980). Fundamentals of Cosmic Physics 5, 287 
Weidner, C., \& Kroupa, P. (2004). MNRAS 348, 187

Weidner, C., \& Kroupa, P. (2005). ApJ 625, 754

Weidner, C., \& Kroupa, P. (2006). MNRAS 365, 1333

Weidner C., Kroupa P., \& Larsen, S. S. (2004). MNRAS 350, 1503

Weidner, C., Kroupa, P., Nürnberger, D., Sterzik, M., (2007). MNRAS, in press

Wuchterl, G., \& Tscharnuter, W. M. (2003). A\&A, 398, 1081

Zinnecker, H. (2003). IAU Symposium 212, 80

Zoccali, M., Cassisi, S., Frogel, J. A., Gould, A., Ortolani, S., Renzini, A., Rich, R. M., \& Stephens, A. W. (2000). ApJ 530, 418

Zoccali, M., et al. (2006). A\&SA 457, L1

\section{Discussion}

TrAGER: Given the variation of the "IGIMF" with galaxy mass,, should high mass galaxies have a flatter IMF than Salpeter?.

KRouPA: Not according to the current formalism of how the IGIMF is calculated: it rests on the assumption that the stellar IMF is invariant, and so the IGIMF $\sim$ IMF if the star-cluster mass function has a power-law index $\beta \leqslant 1.8$, or the IGIMF is steeper than the IMF if $\beta \geqslant 2$.

MEIXNER: So you have passed the buck on the IMF structure to the gas cloud structure. What causes the gas cloud structure?.

KRoupA: It must be turbulence-generated structure, and is probably related to the physical processes acting while the diffuse ISM evolves to a molecular cloud. The recent work by Bonnell et al. on molecular cloud formation in spirals is of importance here.

LAMERS: You predicted that the Orion Nebula cluster has lost already a large fraction of low mass stars due to expansion beyond the tidal radius. But does not the resulting IMF after expansion depend strongly on the adopted initial mass segregation?.

Kroupa: Yes, this is true. We have looked at this in the Pleiades cluster (Moraux et al. 2004), but much work remains to be done on this question. The Pleiades MF appears to be consistent with an initial cluster configuration similar to the very compact Orion Nebula cluster, but not mass-segregated.

DisNEY: How good is the evidence for a correlation for max. stellar and cluster mass?.

Kroupa: The correlation is very good (Weidner \& Kroupa 2006), but it would be important to obtain more data. The correlation is probably a result of feedback termination of star formation in the cluster-forming cloud core: as the cloud core contracts the stellar population builds-up until the cummulative feedback energy reverses cloud collapse, whereby the most massive star would be the dominant energy source (Weidner is currently working on such models.

BECKMAN: I would not like to propose any physical explanation of the stellar IMF, but can give you a reference to a recent paper on the mass function of gas clouds, which yields a curve very comparable to the observations, but uses only simple dynamical considerations, which are independent of metallicity.

KroupA: Yes, thank you. I look forward to seeing this paper (I must have missed it on astro-ph, sorry!. 